\title{
Alterstice
}

Revue internationale de la recherche interculturelle

International Journal of Intercultural Research

Revista International de la Investigacion Intercultural

\section{Les diversités au coeur de la recherche interculturelle : vers de nouvelles perspectives}

\section{Michèle Vatz Laaroussi et Claude Gélinas}

Volume 3, numéro 1, 2013

Les diversités au coeur de la recherche interculturelle : vers de nouvelles perspectives

URI : https://id.erudit.org/iderudit/1077494ar

DOI : https://doi.org/10.7202/1077494ar

Aller au sommaire du numéro

Éditeur(s)

Alterstice

ISSN

1923-919X (numérique)

Découvrir la revue

Citer ce document

Vatz Laaroussi, M. \& Gélinas, C. (2013). Les diversités au coeur de la recherche interculturelle : vers de nouvelles perspectives. Alterstice, 3(1), 3-8.

https://doi.org/10.7202/1077494ar

\section{Résumé de l'article}

Le rapport mondial de l'UNESCO de 2010 souligne que les enjeux autour de la diversité culturelle se sont multipliés et complexifiés en ce début de $\mathrm{XXI}^{\mathrm{e}}$ siècle : en particulier, la reconnaissance des différences et particularités culturelles se heurte à la fois aux dynamiques de mondialisation et de standardisation mais aussi à de nouvelles crispations identitaires et à des replis locaux ou communautaires. Ce sont ces enjeux et ces nouvelles perspectives théoriques, politiques et pratiques autour de la diversité, qu'elle soit culturelle, ethnique, linguistique ou religieuse, qui ont été au coeur des réflexions lors du congrès de l'ARIC (Association internationale de recherche interculturelle) de 2011 et que ce numéro thématique d'Alterstice présente. Comment la recherche interculturelle a-t-elle évolué dans ce contexte ? Sortir de sa routine, voici en quelque sorte ce que l'arrivée du concept de diversité a eu comme effet sur elle : en plus d'ouvrir les frontières disciplinaires et d'articuler de manière originale la théorie et la pratique, le concept de diversité a amené les chercheurs de l'interculturel à s'intéresser aux processus, aux dynamiques et aux transformations en lien avec ces diversités dans des domaines multiples. 


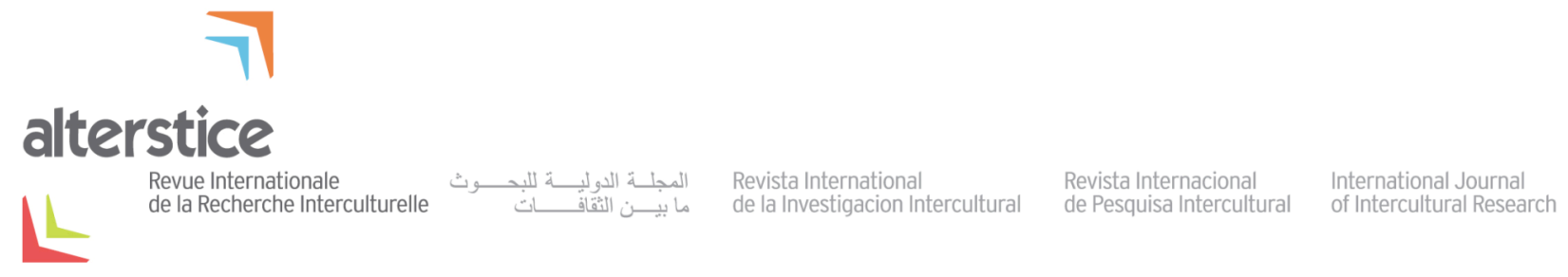

INTRODUCTION THÉMATIQUE

\section{Les diversités au cœur de la recherche interculturelle : vers de nouvelles perspectives}

Michèle Vatz Laaroussi et Claude Gélinas ${ }^{1}$

\section{Résumé}

Le rapport mondial de I'UNESCO de 2010 souligne que les enjeux autour de la diversité culturelle se sont multipliés et complexifiés en ce début de $X X X I^{e}$ siècle: en particulier, la reconnaissance des différences et particularités culturelles se heurte à la fois aux dynamiques de mondialisation et de standardisation mais aussi à de nouvelles crispations identitaires et à des replis locaux ou communautaires. Ce sont ces enjeux et ces nouvelles perspectives théoriques, politiques et pratiques autour de la diversité, qu'elle soit culturelle, ethnique, linguistique ou religieuse, qui ont été au cœur des réflexions lors du congrès de I'ARIC (Association internationale de recherche interculturelle) de 2011 et que ce numéro thématique d'Alterstice présente. Comment la recherche interculturelle a-t-elle évolué dans ce contexte? Sortir de sa routine, voici en quelque sorte ce que l'arrivée du concept de diversité a eu comme effet sur elle : en plus d'ouvrir les frontières disciplinaires et d'articuler de manière originale la théorie et la pratique, le concept de diversité a amené les chercheurs de l'interculturel à s'intéresser aux processus, aux dynamiques et aux transformations en lien avec ces diversités dans des domaines multiples.

\section{Rattachement des auteurs \\ ${ }^{1}$ Université de Sherbrooke, Sherbrooke, Canada}

\section{Correspondance}

michele.vatz-laaroussi@usherbrooke.ca

\section{Mots clés}

diversité; Association internationale de recherche interculturelle (ARIC)

\section{Pour citer cet article :}

Vatz Laaroussi, M. et Gélinas, C. (2013). Les diversités au cœur de la recherche interculturelle : vers de nouvelles perspectives. Alterstice, 3(1), 3-8. 


\section{Introduction}

Depuis le début des années 2000, la diversité est à l'ordre du jour et interpelle tant les intellectuels que les décideurs politiques et les professionnels. L'UNESCO, en promulguant en 2001 une décennie consacrée à la promotion de la diversité culturelle, officialise le terme et le redéfinit par les processus qui la permettent, soit le dialogue entre les différentes cultures du monde. La même année, la Conférence des ministres francophones de la Culture valorise la diversité culturelle au sein de la francophonie internationale et précise que "la diversité culturelle constitue l'un des enjeux majeurs du XXI siècle ». En 2010, I'UNESCO marque la fin de cette opération d'envergure par l'année du rapprochement interculturel mais aussi par plusieurs bilans. Son rapport mondial souligne alors que les enjeux autour de la diversité culturelle se sont multipliés et complexifiés en ce début de XXI ${ }^{e}$ siècle: en particulier, la reconnaissance des différences et particularités culturelles se heurte à la fois aux dynamiques de mondialisation et de standardisation mais aussi à de nouvelles crispations identitaires et à des replis locaux ou communautaires (UNESCO, 2010). Ce sont ces enjeux et ces nouvelles perspectives théoriques, politiques et pratiques autour de la diversité, qu'elle soit culturelle, ethnique, linguistique ou religieuse, qui ont été au cœur du congrès de l'ARIC (Association internationale de recherche interculturelle) en juin 2011 à l'Université de Sherbrooke au Québec. En associant diversité et recherche interculturelle, sous le titre « Les diversités au cœur de la recherche interculturelle: harmonies et dissonances", ce congrès a permis de réfléchir aux nouvelles voies suivies par les recherches interculturelles, elles aussi de plus en plus diversifiées tant par leurs objets que par leurs méthodologies et leurs cadres conceptuels.

\section{La diversité en redéfinition}

C'est d'abord la diversité elle-même qui fait l'objet de recherches pour mieux en saisir les frontières, les processus, I'histoire et les enjeux sociaux, politiques ou épistémologiques. Issu des États-Unis, le concept de diversité culturelle a d'abord été associé à un système social et politique multiculturel dans lequel cohabitent de multiples minorités culturelles, linguistiques et religieuses. Dans cette partie du monde, il a aussi été rapidement connecté au domaine du management, de la gestion des relations humaines au sein des entreprises par exemple. Défini par Anissa Djabi (2011) comme "le respect des différences qui ne doivent pas toutes se fondre dans l'universalisme", ce concept va ensuite parcourir le monde, passant par le Canada, où le multiculturalisme en permet aussi le développement, par exemple dans le monde de l'éducation puis dans le champ des pratiques sociales. En Colombie, la diversité culturelle est incluse dans la Constitution dès la fin des années 1990, associant ainsi les minorités amérindiennes, leurs langues et leurs cultures à l'État nation. Dans les sociétés anglo-saxonnes du monde européen, comme la Grande-Bretagne ou l'Allemagne, il est repris dans les années 1980 et continue à se renforcer dans les domaines de l'administration et des affaires tout en gagnant les relations interculturelles entre les citoyens. Comme nous l'avons souligné en introduction, le concept est promu au niveau international par l'UNESCO au début des années 2000, qui définit le monde comme une "civilisation mondiale multiculturelle ". Finalement, en France, du fait de la perspective républicaine et du principe d'égalité qui régit tous les rapports sociaux, la diversité arrive plus tardivement et va venir compléter l'arsenal juridico-légal concernant la lutte contre les discriminations liées à l'origine nationale, au sexe, à la religion, aux opinions politiques, à l'orientation sexuelle, à l'âge ou au handicap. Dans cette dernière société, on associera dans de nombreuses mesures et institutions, en accord avec les chartes sur les droits et l'égalité, la prévention des discriminations et la promotion de la diversité.

Ainsi, peu à peu, l'histoire de chaque société mais aussi l'existence de rapports internationaux de plus en plus nombreux et complexes ainsi que la multiplication des flux migratoires amènent à redéfinir la diversité, voire à parler de diversités multiples tant au sein d'une même société qu'entre les divers États.

\section{Les domaines contemporains de la diversité}

Ces transformations liées à I'histoire contemporaine et la multiplication des rapports interculturels amènent à reconstruire le paysage de la diversité au travers des différents domaines qu'elle parcourt. Ainsi le monde de l'entreprise privée et des institutions publiques fait maintenant une place importante, et ce, dans divers pays, à la promotion de la diversité culturelle. Vue comme un moyen de refléter la composition réelle de la société dans laquelle ces entreprises se développent, elle est aussi pour plusieurs une manière de prévenir les discriminations.

Alterstice - Revue Internationale de la Recherche Interculturelle, vol. 3, $n^{\circ} 1$ 
Finalement, on la présente comme une valeur ajoutée qui permet un fonctionnement optimal de l'entreprise sur le plan international et dans le cadre des relations à l'interne. Au Québec, plusieurs chercheurs et organisations (voir Ministère de l'immigration et des communautés culturelles, 2007) se sont ainsi intéressés à la promotion de la diversité dans les entreprises comme moyen de mieux intégrer les membres des communautés culturelles. Divers ministères (Proulx, 1998) ont aussi financé des guides permettant de la développer et des documents faisant la promotion des entreprises et institutions qui la mettent en œuvre. En Belgique, on parle du « mainstream de la diversité " (Amoranitis et collab., 2010) comme moyen de faciliter le développement local et la participation citoyenne de toutes et de tous. En France, on va plus loin en créant fin 2004, avec l'aide du ministère de l'Intérieur, la charte de la diversité, qui est signée en 2013 par près de 3500 entreprises et structures privées ou publiques. II s'agit là de promouvoir la diversité dans les recrutements professionnels et les carrières de tous les citoyens, quels que soient leurs origines, leur couleur de peau, leur sexe, leur handicap, leur âge ou leur confession. Le label diversité (Djabi, 2011) vient compléter l'arsenal socio-légal en mesurant et reconnaissant les progrès faits par les employeurs pour prévenir les discriminations et promouvoir la diversité dans leur politique de recrutement et de gestion des ressources humaines. Reconnu par la Communauté européenne comme l'une des meilleures pratiques pour lutter contre les discriminations, ce label diversité fait l'objet de recherches et de diffusion dans plusieurs pays.

En plus de cette lutte contre la discrimination, la diversité, associée dans un premier temps au multiculturalisme, est aussi entrée dans les outils politiques de gestion des relations interculturelles en société, et ce, dans des pays avec des systèmes diversifiés d'intégration. On note l'influence de ce concept de diversité dans les redéfinitions du rôle de l'État et des instances internationales, de la cohésion sociale, de l'intégration et des modèles de gouvernance, créant ainsi des rapports dialectiques entre diversité et citoyenneté (Garcea, 2003).

Touché par ces transformations conceptuelles et politiques de la manière de concevoir le vivre-ensemble tant sur le plan international que national et local, le domaine du droit s'est aussi adapté à ces nouvelles réalités sociales. De plus en plus, on développe une réflexion sur les normes juridiques qui encadrent la différence ethnique, culturelle et religieuse. Le droit de l'immigration, le droit international et les droits de la personne, en particulier, sont revus à la lumière de la diversité et participent par là même au changement des sociétés et des mentalités.

Les paysages urbains se voient reconfigurés par la diversité culturelle et religieuse. Les chercheurs montrent bien comment les métropoles représentent la diversité des sociétés contemporaines et les municipalités mettent ellesmêmes en œuvre des politiques de la diversité qui visent l'intégration de tous les citoyens et l'enrichissement local par cette diversité (Vatz Laaroussi, 2012). Si, dans certains pays, on craint la création de ghettos ethniques, dans d'autres, on valorise les quartiers ou l'entreprenariat ethniques (Ramangalahy et collab., 2009).

De même, les pratiques sociales et professionnelles sont l'objet de transformations à l'aulne de la diversité : on parle de travail social interculturel, d'éducation à la diversité, d'ethnopsychiatrie, ou encore de médiations interculturelles dans le domaine de la santé. On reconnaît que les différences culturelles peuvent avoir un impact dans le rapport à la santé ou encore dans les pratiques autour de la mort. On aborde ces différences non plus en termes de problèmes ou de polarisations tradition-modernité mais plutôt dans une perspective globale et comme relevant des dimensions de l'altérité.

Le domaine religieux est aussi parcouru par la dynamique de la diversité. On parle de pluralisme religieux, on reconnaît les différentes confessions religieuses et la liberté de religion. De plus en plus, on se confronte aussi à la différence religieuse, qu'elle se manifeste dans les rituels, dans les organisations religieuses, dans l'architecture des espaces sacrés, dans l'apparence des fidèles ou encore dans les relations sociales (Gélinas et Derocher, 2012). Dans nos différentes sociétés et sur le plan international, la diversité religieuse est l'un des domaines les plus importants de frottement et de conflits, tout autant qu'elle représente ou peut représenter un espace de rapprochement et de dialogue interculturel.

Bien évidemment, toutes ces transformations sont relayées par les vecteurs sociaux majeurs que sont les médias. Parfois, ils participent à la promotion sociale de la diversité, par exemple en en démontrant la valeur ajoutée pour la société ou en illustrant des parcours réussis de personnes issues des minorités culturelles, linguistiques ou 
religieuses. Parfois, ils participent au contraire à la construction des polémiques et des débats autour de la diversité en laissant une large place à ses détracteurs. Ce fut par exemple le cas lors de la crise des accommodements raisonnables en 2007 au Québec (Potvin, 2008). Les débats sur la diversité ont renvoyé les citoyens à des questionnements sur l'identité nationale, sur leurs spécificités et particularités. Ce sont d'ailleurs les mêmes processus qui sont en jeu en Suisse lors de la médiatisation des référendums sur la construction des minarets.

\section{Sortir la recherche interculturelle des sentiers battus}

Face à tous ces domaines parcourus par la diversité et les processus interculturels qui y sont associés, la recherche interculturelle s'est aussi transformée. Elle a dû développer de nouvelles approches pour déceler ces processus, les comprendre, les analyser. Il lui a fallu diversifier ses disciplines de base, ses fondements, ses perspectives et ses méthodologies.

On a vu se développer la recherche interculturelle qualitative, la recherche interculturelle antiraciste, la recherche interculturelle militante, la recherche-action, en plus des approches empiriques, ethnologiques, anthropologiques, psychologiques et sociologiques. Les sciences juridiques, historiques et politiques sont entrées dans le jeu et, pour observer les diversités, on a inventé de nouvelles lunettes qui font avancer les sciences tout comme les sociétés. De nouveaux cadres conceptuels ont été créés et des méthodologies originales ont vu le jour, portant par exemple sur l'analyse des réseaux internationaux ou sur l'ethnologie urbaine.

Quant aux praticiens, décideurs politiques et gestionnaires, ils ont aussi participé au développement de la recherche sur les diversités en expérimentant de nouvelles approches et en définissant de nouveaux champs d'action. Par exemple, les praticiens du social ont participé au développement de nouvelles méthodologies d'action communautaire interculturelle basées sur l'empowerment et la reconnaissance des identités culturelles. Le monde de la formation aux langues s'est rénové en s'ouvrant à des méthodologies nouvelles comme la biographie langagière ou à des cadres théoriques originaux comme la mobilité linguistique.

Sortir la recherche interculturelle de sa routine, voici en quelque sorte ce que l'arrivée du concept de diversité a produit dans le champ de la recherche. En plus d'ouvrir les frontières disciplinaires et d'articuler de manière originale la théorie et la pratique, le concept de diversité a amené les chercheurs de l'interculturel à s'intéresser aux processus, aux dynamiques et aux transformations en lien avec ces diversités dans des domaines multiples. Sortant du culturalisme, ils ont dû approcher les diversités sous l'angle des rapports sociaux entre individus et groupes en situations inégales, avec leurs particularités, leurs différences mais aussi avec de nécessaires connexions et autour de collaborations et de projets communs.

Ces approches ont permis le développement de nouvelles perspectives. Les concepts et théories colorés par cette perspective sont notamment reliés aux flux migratoires en multiplication et en transformation, à la volonté de conserver le patrimoine culturel de l'humanité, à la mouvance des frontières, à la perméabilité voire à la remise en question des États nations, aux nouvelles conceptions du vivre-ensemble et de la citoyenneté dans des contextes inter-groupes. Ont été développées des recherches et des études sur les familles et les réseaux transnationaux dans les dynamiques migratoires, sur les transformations des normes juridiques, sur la transmission dans les situations de changement culturel, sur la résilience des populations de diverses communautés et minorités, sur la transformation des rapports sociaux ou encore sur les empreintes du religieux dans les paysages urbains.

\section{Des approches multiples pour mieux comprendre la diversité}

Les textes choisis pour ce numéro reprennent des présentations faites au congrès de l'ARIC de 2011. Ils illustrent la multiplicité des objets et des approches possibles lorsqu'on conjugue recherche interculturelle et diversité. Ils viennent aussi enrichir l'arsenal conceptuel et méthodologique autour de la diversité et des rapports interculturels.

Le premier texte correspond à la conférence d'ouverture. II a été rédigé par Michelle Rivet, juriste, première présidente du Tribunal des droits de la personne au Québec et présidente d’honneur du congrès de 2011. Elle introduit la réflexion sur la diversité en dressant le cadre juridico-légal et historique de la notion et de ses usages. En l'abordant tant par le droit international et les chartes qui le parcourent que par le droit national et dans le 
contexte juridico-légal québécois, elle démontre à la fois les énormes potentiels de ce concept mais aussi ses limites et les contradictions qu'il soulève. En particulier, un enjeu majeur de nos sociétés et des futurs rapports internationaux est de faire cohabiter la reconnaissance de la diversité et l'universalité des droits. Michelle Rivet ouvre des voies pertinentes pour ce faire et pour, comme le souligne aussi le rapport de l'UNESCO (2010), mener vers de nouveaux dialogues interculturels et vers la paix.

Gail Mummert, anthropologue et chercheure au Mexique depuis de nombreuses années, continue sur la voie des droits mais les observe, cette fois, sous l'angle très original des familles transnationales. À partir de quatre cas touchant le droit international, les politiques migratoires, les réseaux transnationaux et le lien familial, elle offre une analyse des débats entourant ces situations. Cela permet ainsi de saisir l'importance du concept de familles transnationales, dont l'opérationnalisation peut amener à développer des pratiques visant la reconnaissance des droits des enfants. Elle démontre aussi que ces réseaux transnationaux traversent les frontières des États nations et les remettent en question, amenant de nouvelles pistes pour lier droits, migrations et familles.

Dans la suite de cette réflexion, Annick Germain et Frédéric Dejean, sociologues montréalais, abordent les territoires de la diversité au travers de la multiplication des différents lieux de culte dans les métropoles canadiennes. Ils s'intéressent ici aux débats et controverses qui accompagnent ces nouvelles dynamiques locales et les analysent à la lumière des concepts d'altérité, de transactions et de tissu social. Le religieux, en devenant une expérience urbaine, met au défi de la diversité les municipalités mais aussi de multiples acteurs sociaux. Le développement urbain devient alors un catalyseur de la gestion de la diversité.

Colette Sabatier, psychologue française, est spécialiste des processus d'acculturation, qu'elle analyse et mesure depuis de nombreuses années soit dans des contextes de migration, soit dans des sociétés en contact. Elle nous emmène ici du côté des familles et synthétise cinq recherches de grande envergure qu'elle a menées. Elle approfondit les questions de transmission culturelle et de socialisation des enfants de migrants et des groupes minoritaires en situation de diversité culturelle. Elle démontre que la transmission est un processus complexe, impliquant la participation de plusieurs acteurs en interaction avec la société et ses institutions. Si transmission il y a, elle n'est pas linéaire et elle vise à la fois la culture d’origine et la société d'accueil. Ainsi, elle démontre qu'il y a une transmission des processus d'acculturation et d'adaptation avec une différenciation des rôles parentaux.

Pour terminer, Cécile Rousseau, psychiatre et responsable d'équipes de recherche et d'intervention multidisciplinaires travaillant avec des enfants immigrants et réfugiés au Québec, s'intéresse à l'intervention sociale auprès de ces populations, et plus spécifiquement aux programmes de prévention qui leur sont destinés. Son texte relance le débat autour de l'utilité et des limites de l’approche proposée par les données probantes. Elle offre une réflexion sur le travail avec les enfants immigrants et réfugiés en tant que porteurs de différences. La reconnaissance de la diversité remet en question, selon elle, les savoirs experts et met en évidence la violence possible des processus de normalisation et de standardisation qu'ils ont tendance à promouvoir.

$* * *$

Ces textes démontrent la richesse et l'étendue des recherches interculturelles et la nécessité d'une réflexion critique et éthique. Ils illustrent les défis rencontrés par des recherches s'intéressant à la diversité culturelle et aux rapports sociaux et humains qui la parcourent. Il s'agit de s'ouvrir à une complexité qui tienne compte de la singularité des sujets, des rapports sociaux et des forces collectives tant dans les processus d'intervention auprès des acteurs de la diversité que dans l'analyse qu'on mène des dynamiques qu'ils construisent et produisent. Et il s'agit aussi finalement de garder toujours une attitude de doute, une ouverture à l'incertitude et à la déconstruction! 


\section{Références bibliographiques}

Amoranitis, S., Crutzen, D., Godfroid, J., Manço, A., Partoune, C. et Sensi, D. (2010). Développer le mainstream de la diversité. Recueil analytique d'outils d'intervention pour la valorisation des diversités. Liège / Paris : IRFAM.

Djabi, A. (2011). Le label diversité, un levier pour la prévention et la lutte contre les discriminations. Guide pour l'accompagnement des entreprises candidates au label diversité. Paris : Association française des managers de la diversité (AFMD). www.afmd.fr/IMG/pdf/Guide_Label.pdf

Garcea, J. (2003). L'élaboration et la constitutionnalisation du régime canadien de citoyenneté : une conciliation de la diversité et de l'égalité. Diversité canadienne / Canadian Diversity, 1(3), 118-130.

Gélinas, C. et Derocher, L. (2012). Profil de la diversité religieuse en Estrie. Revue des Cantons de l'Est / Journal of Eastern Townships Studies, 39, 55-72.

Groupe de travail national sur les stratégies relatives aux petits centres (2007). Attirer et garder les immigrants: Une boîte à outils pleine d'idées pour les petits centres ( $2^{\mathrm{e}}$ éd.). Victoria : Inter-Cultural Association of Greater Victoria.

Ministère de l'immigration et des communautés culturelles (MICC) (2007). Miser sur une main-d'œuvre diversifiée: un avantage compétitif pour les PME. Québec: Gouvernement du Québec.

Potvin, M. (2008). Crise des accommodements raisonnables : une fiction médiatique? Montréal : Athéna éditions.

Proulx, J. (1998). Programme de formation: Gestion de la diversité en contexte d'une approche-clientèle et d'une gestion des ressources humaines axées sur les résultats. Sherbrooke : Ministère de la Santé et des Services sociaux / Université de Sherbrooke.

Ramangalahy C., Menzies, T., Filion, L., Brenner, G. et Michel, D. (2009). Entrepreneuriat ethnique et développement local - Comparaisons des contributions des entrepreneurs ethniques au Canada : les Chinois, les Italiens, les Indiens/Sikhs. Montréal : École des hautes études commerciales. (Cahier 2002-08)

UNESCO (2010). Investir dans la diversité culturelle et le dialogue interculturel (Rapport mondial de I'UNESCO n²). Paris : UNESCO. www.unesdoc.unesco.org/images/0018/001847/184755F.pdf

Vatz Laaroussi, M. (2012). Quand la diversité change de visage! Les nouvelles vagues d'immigration et leur impact sur la vision contemporaine de la diversité dans les Cantons de l'Est. Revue des Cantons de l'Est / Journal of Eastern Townships Studies, 39, 9-22.

Alterstice - Revue Internationale de la Recherche Interculturelle, vol. 3, $n^{\circ} 1$ 